\title{
ExoMars Flush Air Data System: Experimental and
}

\section{Numerical Investigation}

\author{
Thorn Schleutker ${ }^{\mathrm{a}}$ and Ali Gülhan ${ }^{\mathrm{b}}$ \\ DLR, German Aerospace Center, 51147 Cologne, Germany \\ Bart Van Hove ${ }^{\mathrm{c}}$ and Özgür Karatekin ${ }^{\mathrm{d}}$ \\ Royal Observatory of Belgium, 1180 Brussel, Belgium
}

The atmospheric reconstruction based on a flush air data system requires knowledge of the surface pressure distribution, which depends on various factors such as the Mach number, angle of attack and aeroshell geometry of the entry vehicle. The purpose of this work is to provide this information for the post-flight analysis of the Entry, Descent and Landing Module of ExoMars 2016, called Schiaparelli. Because the flow around the capsule cannot be duplicated exactly in ground testing facilities, and to account for the uncertainties in physical modelling by numerical simulation, a hybrid approach is chosen for the study. First, the atmospheric entry is simulated experimentally and these experiments are then simulated numerically. The experimental and numerical results are compared and discussed. The almost perfect agreement validates the numerical tool, which afterwards is applied to the analysis of the entry flight of Schiaparelli. A focus lies on the understanding of experimental uncertainties and on the impact of necessary numerical simplifications.

\footnotetext{
${ }^{a}$ Research Scientist, Supersonic and Hypersonic Technology Department, Institute of Aerodynamics and Flow Technologies, Linder Höhe.

b Head of Department and PI of the ExoMars 2016 instrumentation COMARS+, Supersonic and Hypersonic Technologies Department, Institute of Aerodynamics and Flow Technology, Linder Höhe.

c Assistant Researcher, Department of Reference Systems and Planetology, Ringlaan 3.

d Senior Scientist, Department of Reference Systems and Planetology, Ringlaan 3.
} 


\section{Nomenclature}

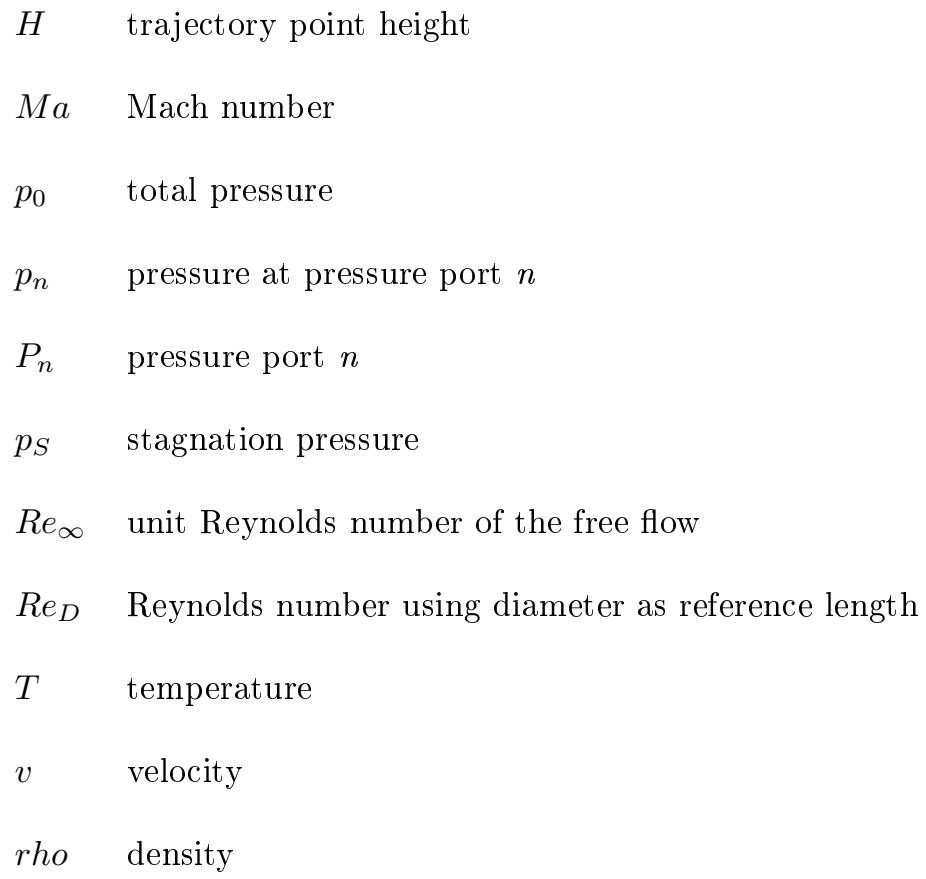

\section{Introduction}

The main objective of the ExoMars program is the exploration of past and present life on Mars. The European Space Agency (ESA) and its partner Russian Space Agency (ROSCOSMOS) decided 
to split it into two separate missions. The first mission ExoMars 2016 consists of deploying the Trace Gas Orbiter (TGO) into the orbit of Mars and demonstrating the entry, descent and landing (EDL) technologies by a successful flight of the Entry, Descent and Landing Module (EDM) Schiaparelli. The second mission ExoMars 2020 aims to land the first European rover on Mars for the in situ investigation of the surface. Besides the demonstration of EDL technologies, Schiaparelli carries instrumentation for collecting scientific data on aerothermal heating and reconstruction of local atmospheric conditions.

The aerothermal design and sizing of the thermal protection system (TPS) of such capsules are carried out using computational fluid dynamics (CFD) codes and ablative material response tools, which are supported by ground experiments. Although ground testing facilities are essential for the validation of these tools, they can cover only a part of the flight environment. Therefore, the physical models in numerical tools can be validated only partially. This results in high safety margins, i.e. high mass in the vehicle design. Assumptions like supercatalytic wall, fully turbulent flow environment, strong roughness induced heat flux augmentation etc. lead to a more than $40 \%$ increase in forebody TPS thickness [1,2]. The TPS mass has a significant impact on the overall mass budget and amount of scientific instrumentation.

To address this, the Mars Science Laboratory (MSL) heat shield was instrumented to acquire important flight data for aerodynamics, aerothermal heating and TPS performance [3, 4]. The MSL sensor package MEDLI (Mars Science Laboratory Entry, Descent, and Landing Instrumentation) allowed collecting pressure, temperature and recession data on the front heat shield [5]. The MEDLI unit comprised of an instrumentation block for thermal performance (temperature and isotherm tracking) of the heat shield called MEDLI Integrated Sensor Plug (MISP) and sensors for surface pressure measurements called the Mars Entry Atmospheric Data System (MEADS). The MEADS pressure sensors are commonly referred to as a Flush Air Data System (FADS). It was implemented to collect aerodynamic data during the supersonic and hypersonic flight regime, by allowing atmospheric density to be reconstructed independently of aerodynamic coefficients.

The conventional approach to density reconstruction is to combine the vehicles speed and acceleration from accelerometer and gyroscope data with predicted aerodynamic force coefficients. Conse- 
quently, the aerodynamic and atmospheric uncertainties are confounded. The vehicle attitude can also be derived from the inertial data, but requires the assumption of zero winds [6]. By contrast, the FADS approach to atmospheric density reconstruction does not rely on assumed aerodynamics and allows separating these uncertainties. Furthermore, the surface pressures are sensitive to wind-relative attitude. Measurements of heat shield surface pressures in the hypersonic and supersonic regime can thus be used to reconstruct atmospheric density and wind-relative attitude, i.e. angle of attack and sideslip. Because atmospheric density from FADS does not depend on assumed aerodynamics coefficients, it can be used also to compute dynamic pressure which is then used to normalize accelerometer force data and reconstruct aerodynamics coefficients. This allows pre-flight aerodynamics models to be validated.

For MSL, several post-flight analyses have demonstrated these FADS capabilities. The pressure measurements have been used to reconstruct the atmospheric density independently of the vehicle acceleration and to derive flight aerodynamics $[7,8]$. The heat shield surface pressure measurements have supported the assessment of the aerodynamic data derived from onboard flight data of inertial-measurement-unit accelerometers and gyroscopes and with the preflight aerodynamic data base. Atmospheric density, pressure and temperature along the entry trajectory of MSL were also reconstructed using heat shield surface pressure data from a single pressure sensor located near the stagnation point [9].

The ExoMars 2016 mission similarly included FADS instrumentation on the front shield for investigation of the aerodynamic performance and for trajectory and atmospheric reconstruction. It consisted of four Kulite pressure sensors of type XTL-DC-123C-190M [10]. The sensors were located on the symmetry axis and at three points on a circle centered on this axis on the front shield of the Schiaparelli capsule [10]. Reconstructing density and attitude from heat shield pressure data requires an accurate model of the heat shield surface pressure distribution [6, 11]. Providing the data for such a model of the ExoMars EDM is the objective of this paper. Subsequently, the study combines experimental investigations on a scaled model, the numerical simulation of these experiments for validation of the numerical tool, and the numerical simulation of the forebody pressure distribution during the atmospheric entry of Schiaparelli. The results are used for the reconstruction 
of free stream properties in the second part of the work [12].

The test gas in the hypersonic wind tunnel facility $\mathrm{H} 2 \mathrm{~K}$ is air with total gas temperatures of up to $1100 \mathrm{~K}$. Therefore, the gas properties of the Martian atmosphere, which has a different chemical composition, cannot be reproduced exactly. The results of the wind tunnel experiments are used primarily for the validation of the numerical tool, which is later adapted to the simulation of the entry of Schiaparelli into Mars' atmosphere. Because of the high computational costs involved in calculating chemical reactions, the 3D flow simulations are carried out assuming chemical equilibrium (EQ). The influence of the chemistry is subsequently studied by performing $2 \mathrm{D}$ non-equilibrium (NEQ) simulations and comparing the results. The NEQ computations are performed at zero angle of attack, for which the flow field is axisymmetric and can be reduced to two dimensions. For a detailed numerical study 3D NEQ computations are necessary.

The paper is organized as follows. Section II covers the experimental investigations of a scaled model and the subsequent numerical simulation of these experiments. After the introduction of the wind tunnel and the scaled model of Schiaparelli, the tests and their uncertainties are presented. The numerical simulation is then discussed and compared to the experimental results. Section III covers the simulation of the entry flight of Schiaparelli. Changes to the boundary conditions and settings of the numerical simulations are pointed out and the numerical grids are discussed. Next, the influence of potential errors in the assumptions regarding the turbulence of the flow and the gas composition of the Mars' atmosphere are studied. The uncertainty introduced by assuming chemical EQ is investigated by comparison with axisymmetric 2D NEQ simulations. Section IV summarizes the paper.

\section{Wind Tunnel Experiments}

The experimental investigation of the surface pressure distribution was conducted in the hypersonic wind tunnel $\mathrm{H} 2 \mathrm{~K}$ of the German Aerospace Centre ( DLR). This wind tunnel was chosen because of its ability to provide hypersonic flows with high flow quality using axisymmetric de Laval nozzles [13]. The lower stagnation temperatures compared to flight and the use of air as test gas prevent the exact reproduction of chemical reactions and the heat capacity ratio as they occur in Mars entry. However, the Mach and Reynolds numbers in the wind tunnel match the flight conditions. 




(a)

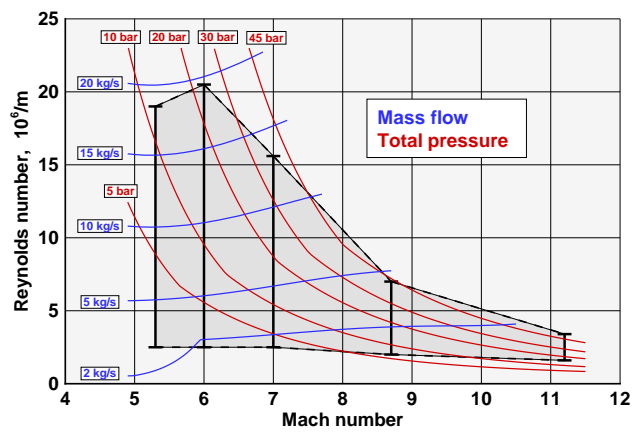

(b)

Fig. 1: Hypersonic Wind Tunnel H2K. Schematical drawing (a) and performance map (b). Source:

[13].

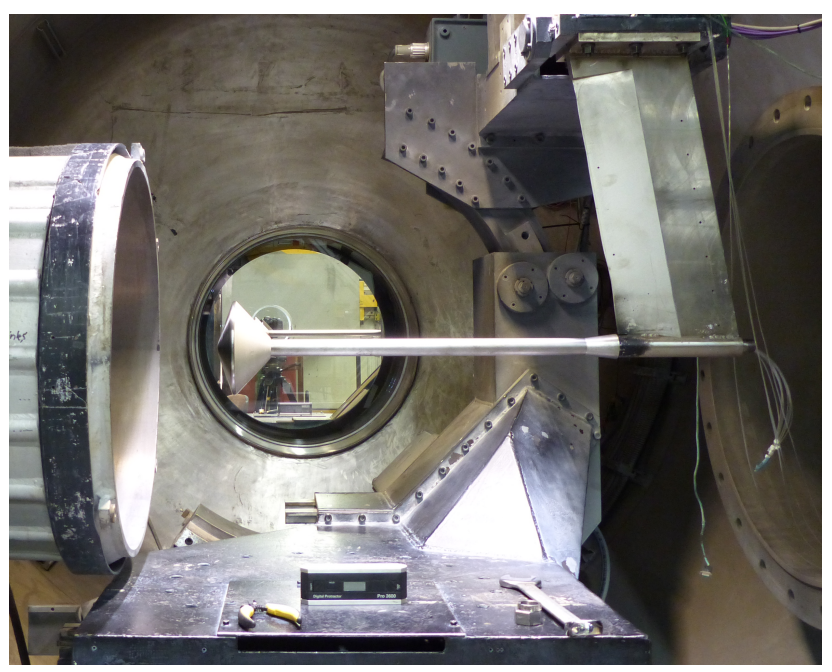

Fig. 2: Test set-up in H2K during installation.

\section{A. Wind Tunnel}

The H2K is a blow down wind tunnel with a free stream test section (Fig. 1a). To allow the flow to build up and stay established over a long testing time, the test chamber is connected to a large vacuum sphere via the diffusor. This sphere is typically evacuated to pressures in the range of a few millibars. The finite volume of $2000 \mathrm{~m}^{3}$ allows for intermittent operation with testing times of around $30 \mathrm{~s}$, depending on the test conditions. The only available test gas is dry air.

Electrical heaters with a power of $5 \mathrm{MW}$ integrated upstream of the nozzle allow heating of the test gas to temperatures of up to $1100 \mathrm{~K}$. This allows adjustment of the stagnation temperature and is also necessary to prevent condensation of the test gas at high Mach numbers. Before the test, 


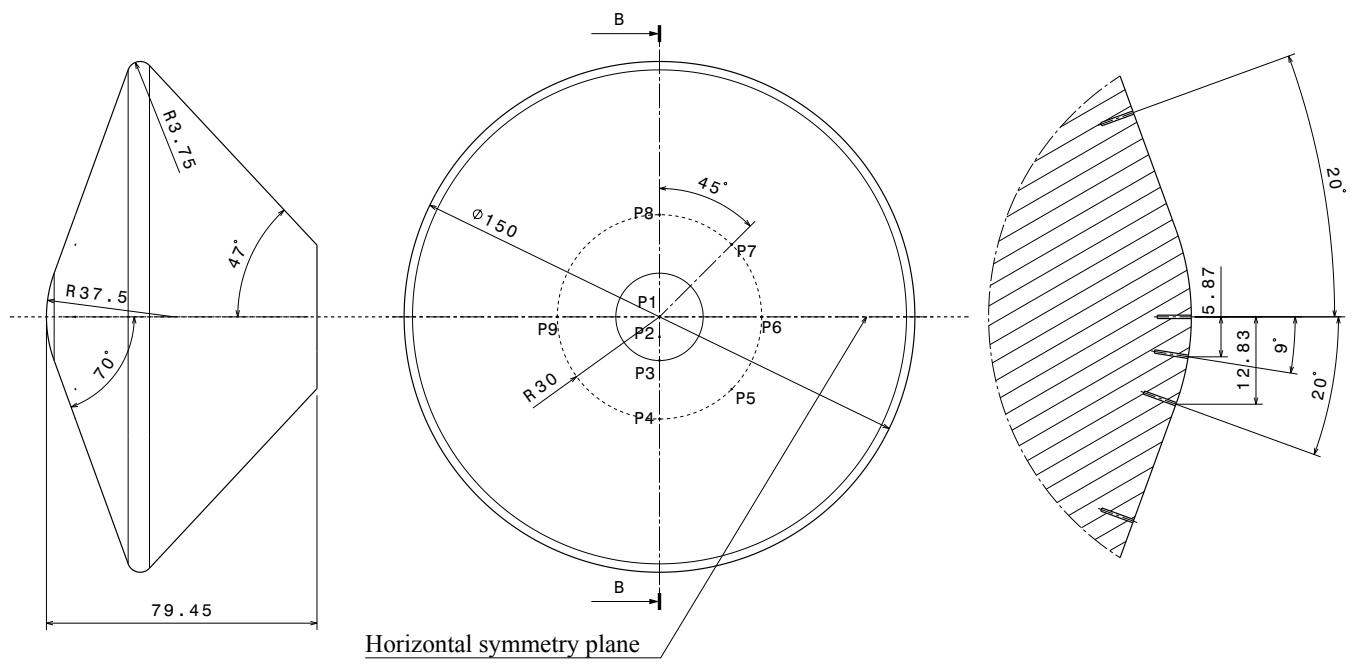

Fig. 3: Drawing of the wind tunnel model with all pressure ports, viewed from the side (left), from the front (middle) and a detail of cut BB (right).

the air flow is channelled through the electrical heaters and released into the atmosphere. Once the heaters have reached steady state at the desired total temperature, a $3 / 2$-way valve is activated to redirect the flow through the nozzle and the test chamber, and finally into the vacuum tank. This procedure ensures maximum test duration, independent of how long the heaters take to reach steady state conditions.

The nozzle geometry and the constant ratio of specific heats of the test gas determine the the Mach number of the flow. The wind tunnel can be equipped with six different contoured nozzles that provide Mach numbers of 5.3, 6.0, 7.0, 8.7 and 11.2. All nozzles have an exit diameter of $600 \mathrm{~mm}$. There is also a Mach 4.8 nozzle, whose nozzle exit diameter is limited to $360 \mathrm{~mm}$. By varying the total pressure and temperature, the Reynolds number can be adjusted between $2.0 \cdot 10^{6} \mathrm{~m}^{-1}$ and $20 \cdot 10^{6} \mathrm{~m}^{-1}$. The range of achievable Mach and Reynolds number combinations is schematically shown in Fig. 1b. Further information about the wind tunnel is provided in [13].

The Schiaparelli model is fixed to the H2K model support arm by a sting attached to the rear of the model. The angle of attack (AoA) is measured with a high-precision angle gage. The experimental set-up is shown in Fig. 2. 


\section{B. Model}

The objective of the wind tunnel experiments is to provide information about the pressure distribution at varying angles of attack and Mach numbers, for validation of the numerical tool and comparison with flight data gathered by Schiaparelli. A scaled model (1:16) is manufactured for this purpose. The model has a diameter of $150 \mathrm{~mm}$, which was selected to prevent flow blockage in the H2K (Fig. 3). To ensure that the local Mach number equals the nominal Mach number all over the capsule, only the core of the flow, which is not influenced by the compression or expansion effects emanating from the nozzle edge, should be used. This is particularly relevant for blunt models, which combine a high degree of redirection of the flow along with a major loss of total pressure due to the strong detached shock.

The front shield of the model is manufactured from solid steel to ensure thermal and geometrical stability during tests as well as a certain robustness. For measuring the surface pressure distribution, holes with a diameter of $0.5 \mathrm{~mm}$ were drilled into the steel front. The line that connects the pressure port and the pressure gage (PSI ESP-16HD) needs to be as short as possible to achieve a fast response of the measurement equipment. Consequently, the gage is implemented inside the model. This restricts the possible number of pressure measurement positions. A total of nine holes is realized.

The front shield of the Schiaparelli capsule was instrumented with four pressure sensors [6]. One sensor was positioned on the symmetry axis of the probe, the others were equidistantly distributed on a circle around this axis. The wind tunnel model needs to reproduce these sensor locations. The positioning of all pressure ports is shown in Fig. 3. The pressure port $P_{1}$ lies on the symmetry axis. To accurately reconstruct the flow angles using FADS, sufficient angular resolution is required. To achieve this, the pressure sensors $P_{4}$ to $P_{8}$ are spaced by $45^{\circ}$. The vertical symmetry of the flow field for non-zero angles of attack is thereby taken advantage of to reduce the instrumentation to only one half of the model. The additional pressure measurement location $P_{9}$ is added opposite $P_{6}$ to check if the flow is symmetrical. Thus, this sensor provides some information about flow homogeneity and correct orientation of the probe. Pressure port $P_{2}$ is positioned in the symmetry plane and on the spherical nose of the model. It is located at $9^{\circ}$ from the axis on the windward side at the estimated 
Table 1: Wind tunnel test conditions

\begin{tabular}{cccccccc}
\hline \hline Condition & Ma & $\begin{array}{c}\mathrm{Re}_{\infty}, \\
10^{6} \cdot \frac{1}{m}\end{array}$ & $\begin{array}{c}\mathrm{Re}_{\mathrm{D}}, \\
10^{6}\end{array}$ & $\begin{array}{c}\mathrm{T}_{0}, \\
K\end{array}$ & $\begin{array}{c}\mathrm{p}_{0}, \\
\mathrm{hPa}\end{array}$ & $\begin{array}{c}\mathrm{p}_{\mathrm{S}}, \\
\mathrm{hPa}\end{array}$ & $\begin{array}{c}\text { Tests, } \\
\text { I }\end{array}$ \\
\hline II & 5.3 & 5.2 & 0.78 & 545 & 5400 & 265 & $0-6^{\circ}, \pm 9^{\circ}$ \\
III & 5.3 & 16.4 & 2.46 & 430 & 12000 & 590 & $+9^{\circ}$ \\
IV & 6.0 & 5.3 & 0.80 & 550 & 7600 & 227 & $0-6^{\circ}, \pm 9^{\circ}, 1^{\circ}$ \\
\hline \hline
\end{tabular}

position of the stagnation point at the highest angle of attack realized in the experiments. Finally, the pressure port $P_{3}$ is positioned at the sphere-cone-transition on the windward side within the symmetry plane. Please regard the plane indicated as horizontal symmetry plane despite pressure measurement holes $P_{2}$ and $P_{3}$ having no equivalent on the other side of this plane.

\section{Tests}

The test conditions include the Mach numbers 5.3, 6.0 and 7.0 to gather information on the Mach number's influence on the surface pressure distribution in the hypersonic flow regime. The corresponding Reynolds numbers are derived from the entry trajectory, which is presented in the second part of the work [12]. The total temperature $T_{0}$ and total pressure $p_{0}$ necessary for achieving the diameter based Reynolds number $R e_{D}$ at a certain Mach number $M a$ are given in Table 1 . The table also includes the resulting stagnation pressure $p_{S}$ and the unit Reynolds number $R e_{\infty}$ of the free flow. The flow condition II with its higher Reynolds number is foreseen for the quantification of the Reynolds number's influence. This high Reynolds condition has no equivalent in the entry trajectory.

Early numerical simulations of the ExoMars EDM entry show a variation of the angle of attack of roughly between zero and six degrees with peaks up to eight degrees or more, depending on the chosen data set $[6,10]$. This supports the simulated trajectory of the companion paper [12] on which the work is based. Thus, the test conditions include angles of attack varying from zero to six degrees in one-degree steps and additionally nine degrees for all Mach numbers. For obtaining 
knowledge about the flow inhomogeneities and their influence, a repetition of the nine-degree test with the model deflected in the opposite direction (minus nine degree angle of attack) is foreseen. This not only turns but also moves the model in the flow. For assessing the repeatability, the $1^{\circ}$ AoA test is conducted a second time at the end of the test campaign for the Mach numbers 6.0 and 7.0. Furthermore, a second test at $9^{\circ}$ and Mach 5.3 with the higher Reynolds number condition II is included for investigating the Reynolds number's influence. In total, the test matrix contains 30 tests (Table 1).

\section{Investigation of Experimental Uncertainties}

The uncertainties of the measurements can be divided into two types: those having the same impact on all pressure measurement positions during one test, further referred to as stagnation pressure uncertainties, and those having different influence on each measurement position and/or measurement. These either have a systematic impact on the measurement (e.g. incorrect orientation of the model and static flow inhomogeneities; systematic uncertainties), are of a transient nature (e.g. sensor noise and transient flow inhomogeneities) or a combination of both (geometric uncertainties of the pressure measurement holes due to manufacturing uncertainties and abrasion). The systematic errors could be corrected if they were isolated and quantified. They will thus be discussed in more detail. General information about wind tunnel measurement techniques and their uncertainties are available in the common literature, for example by Tropea [14]. The influence of the hole geometry for pressure measurement is covered in detail by Nitsche [15].

\section{Stagnation pressure uncertainties}

The total temperature, reservoir pressure and Mach number uncertainties result in a deviation of the stagnation pressure from nominal conditions. The stagnation pressure uncertainty translates directly to an uncertainty of the surface pressure distribution. The uncertainty can be eliminated by normalizing the measurements, however, this results in relative pressure distributions. The stagnation pressure uncertainty is thus of high interest.

The origin of the measured stagnation pressure uncertainties are the variation of the reservoir pres- 
sure, total temperature and Mach number. While the total temperature and the reservoir pressure influence only the stagnation pressure, the Mach number has an impact on both the stagnation pressure and the relative surface pressure distribution. According to hypersonic theory, at high Mach numbers the relative pressure distribution becomes independent of the Mach number, so that modest variations in the Mach number have negligible effect. The relation between stagnation pressure and Mach number, however, is of higher order and significant.

In $\mathrm{H} 2 \mathrm{~K}$ the total pressure is measured with high precision. The pressure sensors uncertainty is $0.1 \%$ of full scale (70 bar). The calibrated standard deviation is $0.0035 \%$. The effective nozzle geometry defines the Mach number but varies depending on the Reynolds number. The Reynolds number's influence on the Mach number through the boundary layer displacement thickness's influence on the effective geometry is well known and characterized. The total temperature is measured with a thermocouple type $\mathrm{K}$ with tolerance class one $( \pm 1.5 \mathrm{~K})$. The temperature influences the stagnation pressure only indirectly over the Reynold number, so the impact of lower precision of temperature measurement on the stagnation pressures is limited. Thus, a very good agreement of the wind tunnel model surface pressures and theoretical stagnation pressure prediction based on the measured total temperature and total pressure would be expected.

Figure 4 compares the wind tunnel data and the numerical results. The measured pressures are divided by the theoretical stagnation pressure derived from the total pressure and Mach number of each respective test. The Mach number is gathered from the calibration of the Reynolds and Mach number correlation of the nozzle. The numerical data is also normalized by dividing it by the calculated stagnation pressure. The figure reveals that the pressures measured in the experiments vary from the theoretical stagnation pressure expectations. As the pressure variations with variation of the angle of attack are of similar order of magnitude as the stagnation pressure deviation, normalizing the experimental data with the theoretical stagnation pressure is not sufficient.

For assessing the flow quality, please note that the Mach number of the wind tunnel is guaranteed to have an maximum error of $\Delta M a= \pm 0.05$. This is based on past calibration measurements [13] and translates to a stagnation pressure uncertainty of $\Delta p_{s}= \pm 3.9 \%$ at Mach 5.3 and $\Delta p_{s}= \pm 3.1 \%$ at Mach 7.0. The deviation observed in the experiments is below that value and so is the deviation 




Fig. 4: Comparison of the experimental and numerical data showing the stagnation pressure uncertainty at Mach 7.0. The measured pressures divided by the theoretical stagnation pressure (dots) are plotted against the numerical data (solid lines).

of the Mach number.

To resolve this issue, all data shown in this section, that was measured in wind tunnel tests or extracted from the numerical simulations of these experiments, is normalized by the following method unless otherwise mentioned. The pressures measured during one test (and consequently at one Mach number and one angle of attack) are divided by the average of the values measured at $P_{1}$ and $P_{4}$ to $P_{9}$ resulting in the normalized pressure

$$
\tilde{p}_{n}=\frac{7 \cdot p_{n}}{P_{1}+\sum_{i=4}^{9} p_{i}}
$$

The basis for normalization can be derived from different pressure signals and their combinations. A possible approach is to divide all pressures by the one measured at the central sensor. However, the approach with the average of multiple pressures as basis is chosen because taking more sensors into account reduces the noise on the data. The positions $P_{2}$ and $P_{3}$ are left out as they do not have an equivalent on the other side of the horizontal symmetry plane. Thus, regarding these two positions, the approach would deliver different results for positive and negative deflections.

Please note also that in post-flight analysis of FADS measurements, pressure data is combined with a pressure model, where the normalization is based on free-stream dynamic pressure [7]. This permits density to be reconstructed, because such normalization relates the surface pressures to 
Table 2: Measured pressure values for positive and negative deflection of $9^{\circ}$ for all Mach numbers, normalized by equation 1 .

\begin{tabular}{|c|c|c|c|c|c|c|c|c|}
\hline $\mathrm{Ma}$ & AoA & $\tilde{\mathrm{p}}_{1}$ & $\tilde{\mathrm{p}}_{4}{ }^{*}$ & $\tilde{\mathrm{p}}_{5}{ }^{*}$ & $\tilde{\mathrm{p}}_{6}$ & $\tilde{\mathrm{p}}_{7}{ }^{*}$ & $\tilde{\mathrm{p}}_{8}{ }^{*}$ & $\tilde{\mathrm{p}}_{9}$ \\
\hline \multirow{3}{*}{5.3} & $-9^{\circ}$ & 1.0288 & 1.0630 & 1.0419 & 0.9903 & 0.9492 & 0.9352 & 0.9917 \\
\hline & $+9^{\circ}$ & 1.0282 & 1.0621 & 1.0446 & 0.9918 & 0.9499 & 0.9359 & 0.9875 \\
\hline & $\Delta$ & $-0.06 \%$ & $-0.09 \%$ & $+0.26 \%$ & $+0.15 \%$ & $+0.07 \%$ & $+0.08 \%$ & $-0.42 \%$ \\
\hline \multirow{3}{*}{6.0} & $-9^{\circ}$ & 1.0345 & 1.0683 & 1.0396 & 0.9801 & 0.9518 & 0.9375 & 0.9882 \\
\hline & $+9^{\circ}$ & 1.0300 & 1.0650 & 1.0395 & 0.9875 & 0.9514 & 0.9370 & 0.9897 \\
\hline & $\Delta$ & $-0.44 \%$ & $-0.32 \%$ & $-0.01 \%$ & $+0.75 \%$ & $-0.03 \%$ & $-0.05 \%$ & $+0.16 \%$ \\
\hline \multirow{3}{*}{7.0} & $-9^{\circ}$ & 1.0305 & 1.0659 & 1.0417 & 0.9884 & 0.9472 & 0.9383 & 0.9881 \\
\hline & $+9^{\circ}$ & 1.0331 & 1.0642 & 1.0400 & 0.9854 & 0.9450 & 0.9418 & 0.9905 \\
\hline & $\Delta$ & $+0.26 \%$ & $-0.15 \%$ & $-0.16 \%$ & $-0.30 \%$ & $-0.23 \%$ & $+0.37 \%$ & $+0.24 \%$ \\
\hline
\end{tabular}

${ }^{*}$ Values at the location pairs P4/P8 and P5/P7 switched for the negative deflection test $\left(-9^{\circ}\right.$ AoA).

free-stream conditions before the shock wave. The normalizations used in this paper are based on a combination of surface pressure data (this section, equation 1) or calculated stagnation pressure (section III, equation 2). Therefore, only relative pressure distributions are validated here when comparing experimental results with numerical modelling. The dependence of stagnation pressure on dynamic pressure is well-known and will be considered in the application of the surface pressure model in [12].

\section{Systematic uncertainties}

To get some knowledge about the flow homogeneity, the $9^{\circ}$ test is repeated with deflection in the opposite direction $\left(-9^{\circ} \mathrm{AoA}\right)$ for all three Mach numbers. The axis, which the model is rotated about, lies behind the rear of the model (179 $\mathrm{mm}$ from the nose). This implies, that the model is moved perpendicular to the flow when rotated and thus is exposed to a different part of the flow field. However, the movement orthogonal to the flow axis is rather small $(28 \mathrm{~mm})$ and the effect is overlaid by the influence of uncertainties from the imperfect geometry. The normalized pressures measured within these tests are presented in Table 2.

The pressure measurement locations outside the horizontal symmetry plane are distributed over the 
Table 3: Measured pressure data normalized by equation 1 showing the repeatability at Mach 6.0 and Mach 7.0.

\begin{tabular}{ccccccccccc}
\hline \hline Ma & AoA & $\tilde{\mathrm{p}}_{1}$ & $\tilde{\mathrm{p}}_{2}$ & $\tilde{\mathrm{p}}_{3}$ & $\tilde{\mathrm{p}}_{4}$ & $\tilde{\mathrm{p}}_{5}$ & $\tilde{\mathrm{p}}_{6}$ & $\tilde{\mathrm{p}}_{7}$ & $\tilde{\mathrm{p}}_{8}$ & $\tilde{\mathrm{p}}_{9}$ \\
\hline & $+1^{\circ}$, Test 1 & 1.0489 & 1.0463 & 1.0326 & 1.0009 & 0.9952 & 0.9867 & 0.9919 & 0.9841 & 0.9923 \\
6.0 & $+1^{\circ}$, Test 2 & 1.0489 & 1.0460 & 1.0320 & 1.0008 & 0.9949 & 0.9857 & 0.9921 & 0.9845 & 0.9930 \\
& \multicolumn{1}{c}{$\Delta$} & $+0.00 \%$ & $-0.03 \%$ & $-0.06 \%$ & $+0.00 \%$ & $-0.03 \%$ & $-0.10 \%$ & $+0.02 \%$ & $+0.04 \%$ & $+0.07 \%$ \\
\hline & $+1^{\circ}$, Test 1 & 1.0455 & 1.0419 & 1.0278 & 0.9969 & 0.9962 & 0.9937 & 0.9856 & 0.9870 & 0.9950 \\
7.0 & $+1^{\circ}$, Test 2 & 1.0451 & 1.0412 & 1.0272 & 0.9965 & 0.9983 & 0.9962 & 0.9867 & 0.9868 & 0.9903 \\
\hline \hline & $-0.04 \%$ & $-0.07 \%$ & $-0.06 \%$ & $-0.04 \%$ & $+0.21 \%$ & $+0.25 \%$ & $+0.11 \%$ & $-0.02 \%$ & $-0.47 \%$ \\
\hline \hline
\end{tabular}

models front surface symmetrically except for the points $P_{2}$ and $P_{3}$, which have no equivalent on the other side. This symmetry allows comparing the results for the same angle of attack but with different deflection directions if the axis of rotation lies in the horizontal symmetry plane, which is the case. Consequently, the test results with different deflection directions can be compared directly by exchanging the measured pressure values at the locations $P_{4} / P_{8}$ and $P_{5} / P_{7}$ for one of the tests. In Table 2, the values at these locations are switched in case of the deflection in negative direction. The locations $P_{2}$ and $P_{3}$ are not of interest for this comparison because of the missing equivalents on the other side of the horizontal symmetry plane and thus disregarded. The columns with $\Delta$ as angle of attack present the relative difference $\Delta=\left(\tilde{p}_{+9^{\circ}}-\tilde{p}_{-9^{\circ}}\right)$ between the pressures at positive and negative deflections. The table shows a very good agreement of the measured pressure distributions. The differences between the relative pressures measured for different deflection directions are in general below half a percent, except for Mach 6.0, were the difference at location $P_{6}$ is higher. The table reveals no persistent systematic errors, which could be caused by incorrect pressure port positions or non-symmetrical geometry and would show a similar deviation for all Mach numbers at one pressure port.

The repeatability is studied with a second test at $1^{\circ}$ AoA for Mach 6.0 and Mach 7.0. The original test and the repetition were performed under exactly the same conditions but on different test days. The results are displayed in Table 3 . The results of the original and the replicated test agree almost perfectly for Mach 6.0. The deviation is higher at Mach 7.0, but still very good. $P_{9}$ is the only 
Table 4: Test results in comparison for different Reynolds numbers (flow condition I and II) at Mach 5,3 and $9^{\circ}$ Angle of Attack.

\begin{tabular}{lccc}
\hline \hline & Flow condition I & Flow condition II & Difference \\
\hline $\operatorname{Re}_{\infty}$ & $5.25 \cdot 10^{6}$ & $16.0 \cdot 10^{6}$ & $+204 \%$ \\
$\mathrm{p}_{0}$ & $265 \mathrm{hPa}$ & $571 \mathrm{hPa}$ & $+115 \%$ \\
$\tilde{\mathrm{p}}_{1}$ & 1.0135 & 1.0136 & $+0.02 \%$ \\
$\tilde{\mathrm{p}}_{2}$ & 1.0428 & 1.0435 & $+0.08 \%$ \\
$\tilde{\mathrm{p}}_{3}$ & 1.0576 & $+0.00 \%$ \\
$\tilde{\mathrm{p}}_{4}$ & 1.0576 & 1.0483 & $+0.14 \%$ \\
$\tilde{\mathrm{p}}_{5}$ & 1.0468 & 1.0295 & $-0.01 \%$ \\
$\tilde{\mathrm{p}}_{6}$ & 1.0296 & 0.9783 & $+0.06 \%$ \\
$\tilde{\mathrm{p}}_{7}$ & 0.9373 & $+0.10 \%$ \\
$\tilde{\mathrm{p}}_{8}$ & 0.9776 & 0.9219 & $-0.05 \%$ \\
$\tilde{\mathrm{p}}_{9}$ & 0.9363 & 0.9700 & $-0.34 \%$ \\
\hline \hline
\end{tabular}

exception. At this position, the deviation of the normalized pressures is close to half a percent. Overall, the repeatability is good and no systematic errors are observed.

\section{Reynolds number}

Quantifying the Reynolds number's influence on the results is important, as the number varies slightly with each test. Thus, one test was carried out at a higher Reynolds number (condition II). For that purpose, the Mach 5.3 and $9^{\circ}$ AoA case was utilized. The results are presented in Table 4. They agree almost perfectly with some outliers with slightly higher deviations with different algebraic signs. Due to the different algebraic signs, these deviations cancel out each other in the average, which is useful for the normalization. This explains the good agreement between the results of tests at different Reynolds numbers. The deviations seem not to be of a systematic nature. A general Reynolds number dependence is not obvious.

\section{E. Numerical Simulations of the Experiments}

The wind tunnel experiments are numerically rebuilt by performing Reynolds-averaged NavierStokes simulations on unstructured grids with DLR's finite volume solver TAU $[16,17]$. Due to 


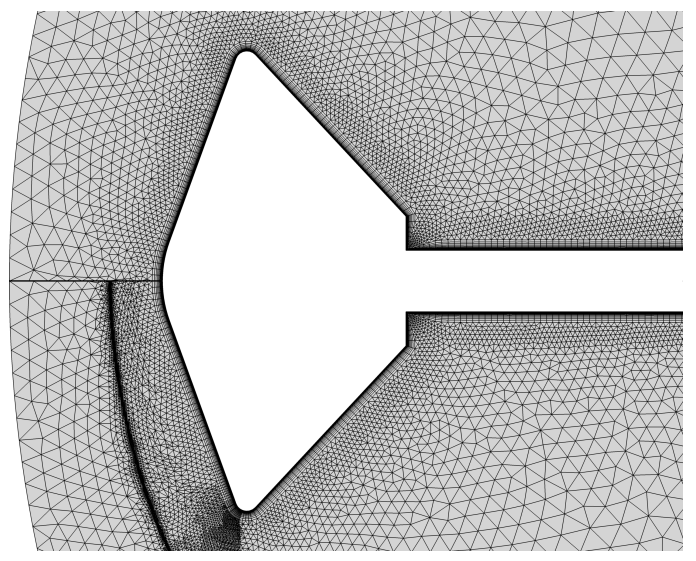

(a) Detail of the grid in the symmetry plane. The limitation of the adaptation routine to the volume before the shoulder is obvious.

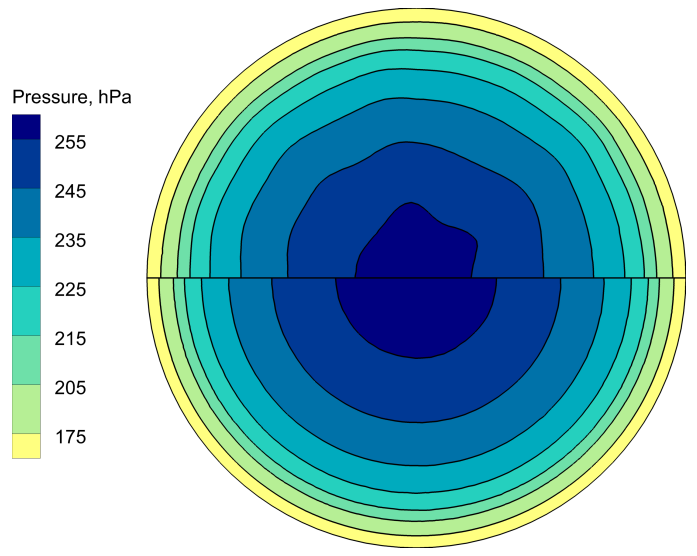

(b) Surface pressure distribution on the front

shield. The importance of the adaptation becomes apparent in the rotational symmetry.

Fig. 5: Images of the numerical simulation of the Mach 5.3 and $0^{\circ}$ AoA experiment after initial calculation (top) and final adaptation (bottom).

the turbulence present in the free stream of the wind tunnel and rather high Reynolds numbers, a completely turbulent flow around the model is assumed. The simulations are carried out on a hybrid grid generated by Centaur [18] with 20 prism layers. These allow a sufficient resolution of the boundary layer and also the adaptation routine of TAU to adjust the thickness of the elements close to the wall to ensure a dimensionless wall distance $y+$ below unity. The shear stress transport turbulence model of Menter [19] is used. A smooth wall with non-slip and adiabatic boundary conditions is assumed. The convergence is accelerated by multi-grid start-up and local time stepping. The test set-up is modelled including the base assuming stationary flow. The fixation sting with its diameter of $20 \mathrm{~mm}$ is regarded (see Fig. 5a).

The symmetry is utilized for cutting the flow field in half. The initial grid with 1.5 million cells is adapted by TAU during the simulation to achieve a good resolution of the bow shock and the region between shock and front surface. The refinement of the grid is thus limited to the volume in front of the model. The grid grows to a number of 19 million cells. Comparing the results with and without adaptation, it is apparent that this is necessary for accurate surface pressures and a smooth distribution. Figure 5a shows the initial and the adapted grid. An example of the front pressure 


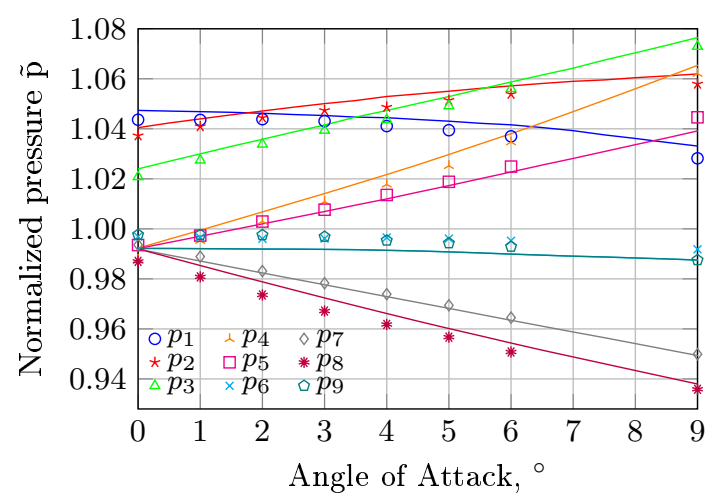

(a) Mach 5.3.

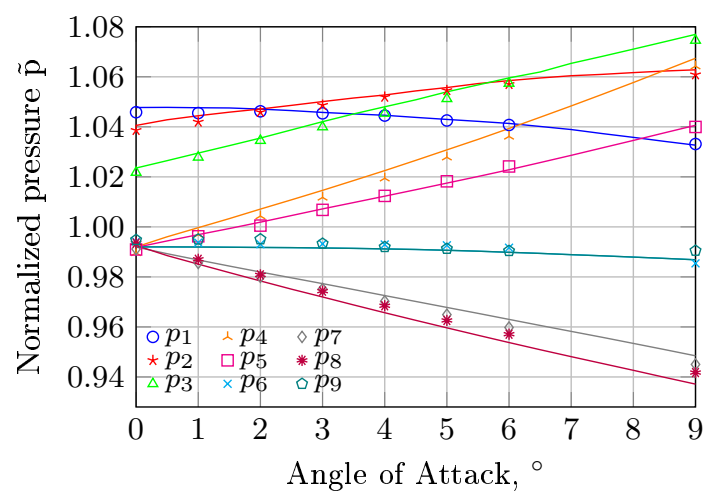

(c) Mach 7.0.

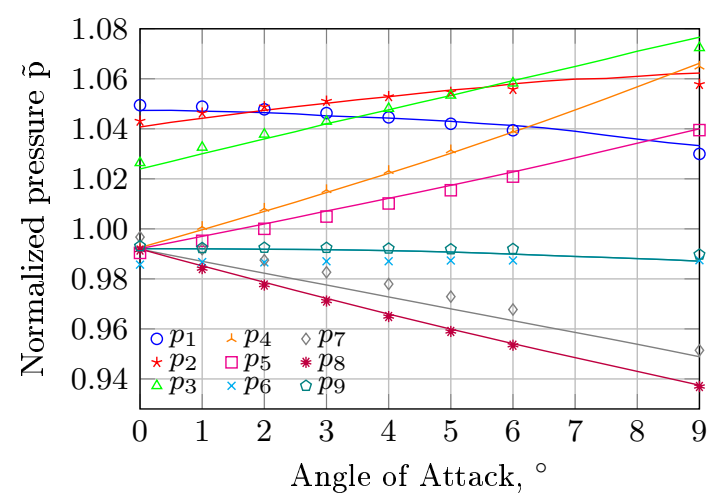

(b) Mach 6.0.

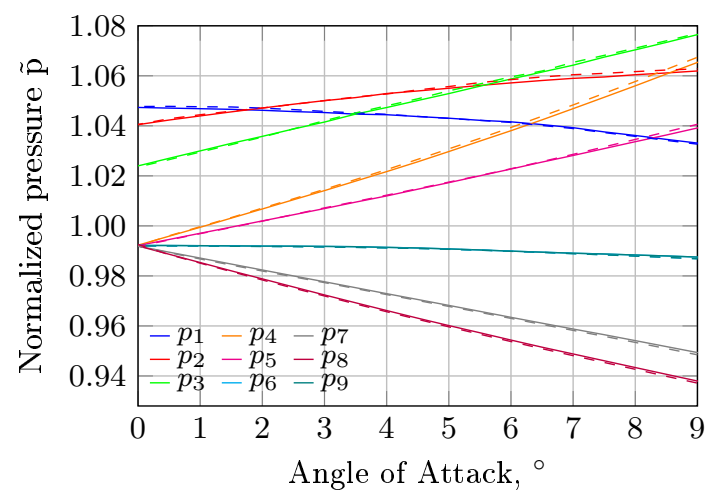

(d) Mach 5.3 (solid) and Mach 7.0 (dashed).

Fig. 6: Comparison of the experimental (dots) and numerical (lines) data at different Mach numbers, all normalized by equation 1 .

distribution is given in Fig. 5b. This figure not only demonstrates the necessity of the adaptation, but also indicates the quality of the $3 \mathrm{D}$ simulation. The $0^{\circ} \mathrm{AoA}$ case is axisymmetric. Therefore, the $3 \mathrm{D}$ solution is supposed to be axisymmetric too, as is the case for the grid after final adaptation.

\section{F. Comparison of Experimental and Numerical Results}

The experimental and numerical results of all three Mach numbers are visualized in Fig. 6. A good agreement between experiments and numerics is found and the differences between the measured and calculated normalized pressures are low. Note that equation 1 is used for normalization. More important, the experimental data almost perfectly agrees with the predicted trend of the pressure over the angle of attack at different pressure ports. For each individual pressure port, the offset of the experimental results with respect to the numerical data is almost constant over the angle of 
attack at a particular Mach number. The pressures at the positions $P_{4}$ to $P_{9}$ are expected to be exactly the same at $0^{\circ}$ AoA. This offers the possibility to correct the offset. However, this would rely on the assumption of perfect angular orientation of the model. The offset would then only result from geometrical imperfections and flow inhomogeneities. As an imperfect angle of attack setting cannot be ruled out, the data is presented without the correction of this offset.

The differences between the measurements at the three Mach numbers are small. Figure 6d highlights this by comparing the numerical Mach 5.3 and Mach 7.0 results. This is consistent with the hypersonic theory, which predicts Mach number independence of the pressure distribution. Calculating the correct Mach number from the measured pressure distribution does not seem to be possible for the wind tunnel data.

In summary, the experimental data confirms the numerical simulations. The validated numerical approach is next used for calculating the pressure distributions at conditions experienced by Schiaparelli during atmospheric entry on Mars.

\section{Atmospheric Entry Simulations}

As the purpose of this work is to provide pressure distributions for flow angle and atmospheric reconstruction using the FADS approach [12], the numerical flow simulations are carried out for flight conditions gathered from a predicted ExoMars EDM entry trajectory, which is is presented in [12], see also [10]. The trajectory points are chosen to be representative and sufficiently dense spaced. The Mach number $M a$ range is from Mach 2.0 to Mach 14.0 and the height $H_{0}$ goes from $9.7 \mathrm{~km}$ to $27 \mathrm{~km}$. Table 5 summarizes the trajectory points. It also provides the values of the velocity of the capsule with respect to the ground $v_{\infty}$, the local atmospheric temperature $T_{\infty}$ and density $\rho_{\infty}$, and the Reynolds number $R e_{D}$. For the numerical investigation of the atmospheric entry, the simulations verified by the wind tunnel data are adapted. Thus, the tools used and most of their settings and parameters remain unchanged. As such, the trajectory points are also simulated with DLR's TAU solver on a grid generated by Centaur.

Several changes to the simulations are implemented because of the different boundary conditions compared to the simulation of the wind tunnel tests. The most obvious changes are the composition of the atmosphere of Mars and the modelling of chemical reactions. The chemistry is important 
Table 5: Trajectory points for numerical simulation.

\begin{tabular}{ccccccc}
\hline \hline TP & Ma & $\mathrm{H}, \mathrm{km}$ & $\mathrm{v}_{\infty}, \frac{\mathrm{m}}{\mathrm{s}}$ & $\mathrm{T}_{\infty}, \mathrm{K}$ & $\rho_{\infty}, \frac{\mathrm{g}}{\mathrm{m}^{3}}$ & Re $_{\mathrm{D}}{ }^{*}$ \\
\hline I & 2.0 & 9.67 & 475.6 & 221.7 & 5.950 & $622 \mathrm{k}$ \\
II & 3.0 & 13.3 & 705.0 & 216.9 & 4.410 & $685 \mathrm{k}$ \\
III & 4.0 & 15.6 & 933.5 & 213.4 & 3.622 & $744 \mathrm{k}$ \\
IV & 5.0 & 17.4 & 1159 & 210.4 & 3.115 & $796 \mathrm{k}$ \\
V & 7.0 & 20.1 & 1607 & 205.8 & 2.490 & $881 \mathrm{k}$ \\
VI & 9.0 & 22.3 & 2046 & 201.7 & 2.055 & $928 \mathrm{k}$ \\
VII & 11.0 & 24.1 & 2482 & 198.0 & 1.746 & $956 \mathrm{k}$ \\
VIII & 14.0 & 27.1 & 3132 & 191.9 & 1.339 & $924 \mathrm{k}$ \\
\hline \hline
\end{tabular}

* Reynolds number based on free-stream conditions, capsule diameter of $2.4 \mathrm{~m}$ and assumed constant viscosity of $10.9 \cdot 10^{-6} \mathrm{Pas}$.

because of the high gas temperatures resulting from the high velocities at the upper trajectory points.

The Mars atmosphere mainly consists of carbon dioxide $\left(\mathrm{CO}_{2}\right)$, but also contains relevant quantities of nitrogen $\left(\mathrm{N}_{2}\right)$ and argon [20]. The most detailed model of the chemical reactions in Mars entry available for TAU at the time of this work consists of 13 species and 103 reactions and was developed in the frame of the SACOMAR project [21]. Argon, however, is not included in this model. The atmosphere composition used for the simulations is $97.2 \% \mathrm{CO}_{2}$ and $2.8 \% \mathrm{~N}_{2}$ for both the $\mathrm{EQ}$ and the NEQ calculations. The influence of the gas composition on the solutions is investigated by altering the composition to $95.0 \% \mathrm{CO}_{2} / 5.0 \% \mathrm{~N}_{2}$ and pure $\mathrm{CO}_{2}$.

At zero degree angle of attack, the flow field around the capsule is axisymmetric and can be reduced to two dimensions. At non-zero angles, this is not possible and the three-dimensional nature of the flow needs to be taken into account. Because of the high computational costs of solving the NEQ equations, it is not possible for the authors to calculate a sufficiently resolved 3D solution for NEQ. To cope with that, EQ is subsequently assumed. To assess the error introduced by this simplification, NEQ calculations are performed on a 2D grid for comparison. A non-reacting gas 
with variable heat capacity and viscosity, which are calculated from the (constant) gas composition and the local temperature, is also considered for comparison. The models used in TAU are described in $[22]$.

Another adaptation is necessary for the thermal boundary conditions of the capsule. The thermal inertia, the radiation of heat, and the endothermic ablation of the heat shield effectively act as a heat sink and dictate a surface temperature below the recovery temperature of the flow resulting in a subsequent heat flux to the surface. Publicly available information on the surface temperature distribution or the magnitude of these effects is not known by the authors. To account for this, radiative EQ is assumed as a best guess. Carbonaceous ablators have emissivities near the physical upper limit when charring, with $\epsilon=0.85$ being a typical value [23]. For the simulations, an emissivity of $\epsilon=1.0$ is assumed. The higher value is chosen because the other heat sink mechanisms are not modelled. Despite this, the resulting surface temperatures are probably too high at the upper trajectory points, where there is significant chemical decomposition and the thermal inertia of the TPS limiting the temperature increase over time. At the lower trajectory points, the neglecting of the thermal inertia and chemical surface reactions potentially leads to an underestimation of the surface temperatures. However, the surface temperatures have low influence on the pressure distribution, as the influence is limited to the thin thermal boundary layer. For the NEQ calculations, the influence of the catalytic activity is also examined, as the real value is unknown. Thus, the NEQ simulations are conducted assuming both a non-catalytic wall and a wall with full catalytic recombination.

Furthermore, a laminar flow is assumed. This is justified by several publications that investigate the laminar-turbulent transition on the front of blunt entry bodies. An overview is provided by Schneider [24]. The consensus is that the flow on the front is laminar at the Reynolds numbers experienced by Schiaparelli during flight, which range from 622 thousand to 956 thousand (see Table 5). The ablation of the heat shield leads to surface roughening and outgassing of pyrolysis products $[25,26]$. While the surface roughness generally can lead to early transition, it is discussed that the gas injection could induce turbulence already at the stagnation point [26]. Therefore, fully turbulent calculations based on the shear-stress transport model of Menter [19] are conducted for comparison. 


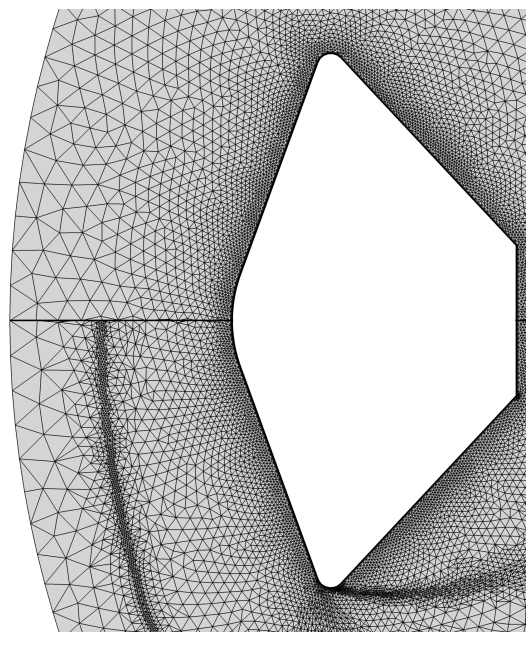

(a) Extended grid.

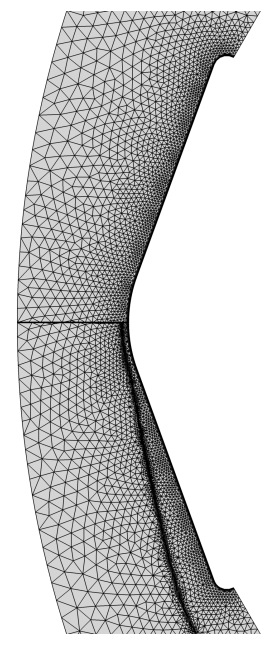

(b) Shortened grid.

Fig. 7: Numerical grids before (top) and after (bottom) final adaptation step at $0^{\circ}$ AoA for trajectory points I (a) and VIII (b).

Please regard that for all pressure data provided in this section, the theoretical stagnation pressure $p_{S}$ is used as basis for normalization:

$$
\hat{p}_{n}=\frac{p_{n}}{p_{S}}
$$

This is because only numerical results are compared for which theoretical stagnation pressure works well. This differs from the normalization approach used in the previous section.

\section{A. Grid Considerations}

The 3D grids used for the numerical simulations of the atmospheric entry differ from the grid used for the simulation of the wind tunnel experiments. The resolution in the proximity of the capsule is increased to sufficiently resolve the transition from the post-shock chemical composition and high temperatures to the conditions near the surface. Furthermore, despite the laminar flow, 17 prism layers are used to resolve the gradients in the vicinity of the surface. The adaptation of the grid is used less intensely to reduce the computational costs, meaning the shock is less resolved. Three different 3D grids are used for the simulations. The basic grid is used for the trajectory points $I I$ to $V$. An adapted grid is required for trajectory point I, as the Mach 2.0 flow requires a thicker 
mesh in front of the capsule due to the increased shock stand-off distance. A third grid is generated to reduce the computational effort at high Mach numbers, where information from the back of the capsule cannot be transported upstream and the flow ahead of the capsule is not influenced by the back-side solution. In this case, it is sufficient to simulate the region upstream the shoulder. The nominal grid is accordingly cut and the resulting third version is used for the upper trajectory points VI to VIII. The nominal grid and the version extended in front of the capsule both start with 2.6 million cells and are refined to 13 million cells during the simulation. The shortened grid has a lower cell count, starting with 1.2 million and increasing to 5.8 million cells.

A fourth grid is necessary for the $2 \mathrm{D}$ calculations. As there is no point in investigating the influence of the chemistry and gas composition at the lower trajectory points, at which the flow is cold and non-reactive flow, a single 2D grid is created based on the nominal grid without extending the mesh in front of the capsule. The resolution of the grid in the proximity of the shock and the wall is crucial for the NEQ simulations and is thus taken to the highest reasonable cell count. The grid has 45 thousand cells when unadapted and grows to 150 thousand cells after the final adaptation step in the NEQ calculations. Besides being used for the chemistry investigation, it is also used for the investigation of the effect of the turbulence model and the gas composition. In these calculations, the computational effort is non-restrictive and the grid is refined up to a cell count of 420 thousand. The number of prism layers is 25 . The extended and the shortened grids are presented in Fig. 7 .

\section{B. Turbulence}

The pressure data of the turbulent simulation coincides almost perfectly with the laminar simulation. The turbulent boundary layer development and velocity distribution differ from the laminar boundary layer. Therefore, a variation of the displacement height as well as the shock stand-off distance can be expected. However, this effect is not observed in the simulations. As the boundary layer is thin compared to the flow dimensions, the difference in the displacement height is too small to be relevant for the shock geometry. Table 6 highlights this by comparing the pressures at locations according to those of the sensor positions on the wind tunnel model for the two solutions. The differences are in the order of the noise level of the data. This is also obvious in Fig. 8, where the surface pressure distribution is plotted. The results of the nominal case (meaning the laminar 
Table 6: Comparison of the normalized pressures for laminar / turbulent conditions and different gas compositions at trajectory point VIII and $0^{\circ}$ Angle of Attack.

\begin{tabular}{lcccc}
\hline \hline Boundary condition & $\hat{\mathrm{p}}_{1}$ & $\hat{\mathrm{p}}_{2}$ & $\hat{\mathrm{p}}_{3}$ & $\hat{\mathrm{p}}_{4}$ \\
\hline Nominal & 1.009 & 0.982 & 0.932 & 0.957 \\
\hline Turbulent & 1.009 & 0.983 & 0.934 & 0.956 \\
& $+0.0 \%$ & $+0.0 \%$ & $+0.2 \%$ & $-0.1 \%$ \\
\hline $95 \% \mathrm{CO}_{2}$ & 1.009 & 0.983 & 0.935 & 0.957 \\
& $-0.0 \%$ & $+0.0 \%$ & $-0.0 \%$ \\
\hline $100 \% \mathrm{CO}_{2}$ & 1.010 & 0.982 & 0.929 & 0.957 \\
& $+0.0 \%$ & $-0.0 \%$ & $-0.3 \%$ & $-0.0 \%$ \\
\hline \hline
\end{tabular}

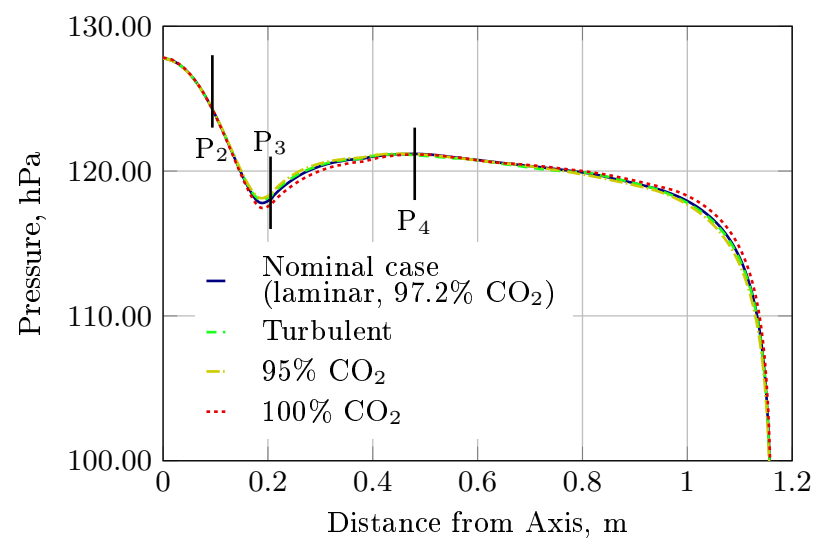

Fig. 8: Pressure distribution on the front surface for the nominal case, turbulent flow and varying gas compositions at trajectory point VIII.

flow case) and the turbulent simulation coincide almost perfectly. The pressure port $P_{3}$ seems to be most sensitive to the flow turbulence. However, no pressure port is situated at this location on the EDM heat shield.

\section{Gas Composition}

The variation of the gas composition in the range from pure carbon dioxide to a mixture with up to five percent nitrogen has low influence on the shock geometry and pressure distribution in case of chemical EQ. Hence, the atmospheric reconstruction's sensitivity to the assumed gas composition is low. The composition does have a small influence that becomes apparent when there is significant 


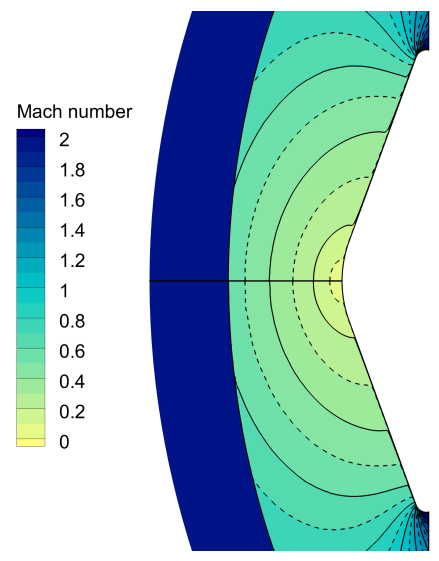

(a)

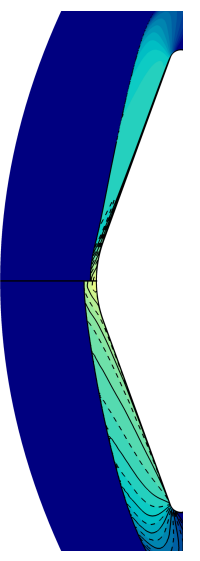

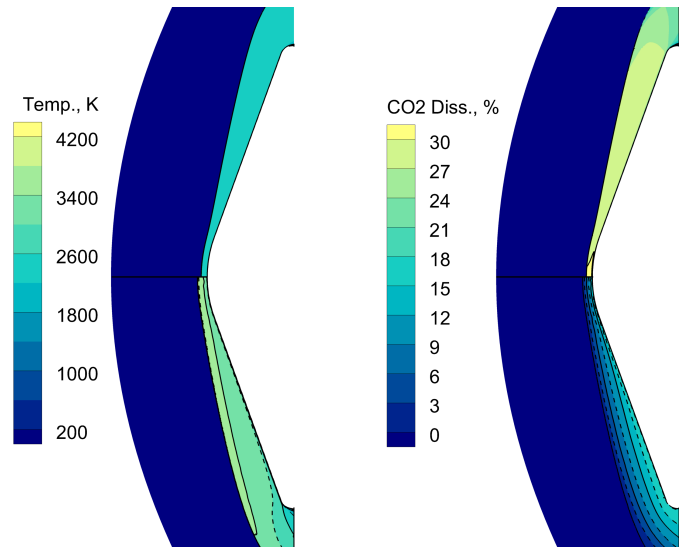

(b)

Fig. 9: Visualisation of results for different chemistry assumptions. Figure (a) highlights the Mach number distribution in a section of the symmetry plane for the EQ gas (top) and non-reacting gas

(bottom) at trajectory point I (left) and VIII (right). Figure (b) provides the temperature distribution (left) and percentage of dissociated $\mathrm{CO}_{2}$ (right) for the chemical EQ (top) and NEQ (bottom) at trajectory point VIII.

chemistry in the flow. The pressure distributions at trajectory point VIII are compared in Table 6 and Fig. 8.

At the upper trajectory points, a higher nitrogen content increases the shock stand-off distance and subsequently varies the pressure distribution slightly due to the chemical behaviour of the atmospheric gas components. This can be seen by investigating the mass fractions at trajectory point VIII. At nominal conditions, the $\mathrm{CO}_{2}$ mass fraction drops from $97.2 \%$ down to a minimum of around $68 \%$. This means that almost $30 \%$ of the carbon dioxide molecules are dissociated. The nitrogen on the other hand shows low dissociation at the temperatures involved. Its mass fraction drops no lower than $2.64 \%$, which translates to a dissociation of only $6 \%$ of the nitrogen. It therefore behaves almost as a non-reacting gas when compared to the carbon dioxide. A higher nitrogen content shifts the simulation results towards those of an non-reacting gas.

\section{Chemistry}

Chemical reactions only occur above certain temperatures. These are quite high for the reactions predominant for the Mars entry, namely the endothermic dissociation of carbon dioxide [20]. 
Table 7: Comparison of the normalized pressures for the assumption of a non-reacting gas, chemical EQ and chemical NEQ at trajectory point VIII and $0^{\circ}$ Angle of Attack.

\begin{tabular}{lcccc}
\hline \hline Boundary condition & $\hat{\mathrm{p}}_{1}$ & $\hat{\mathrm{p}}_{2}$ & $\hat{\mathrm{p}}_{3}$ & $\hat{\mathrm{p}}_{4}$ \\
\hline Nominal & 1.009 & 0.982 & 0.932 & 0.957 \\
\hline Non-reacting & 1.003 & 0.988 & 0.962 & 0.948 \\
& $-0.6 \%$ & $+0.5 \%$ & $+3.2 \%$ & $-0.9 \%$ \\
\hline NEQ, non-catalytic & 0.999 & 0.981 & 0.955 & 0.951 \\
& $-1.0 \%$ & $-0.1 \%$ & $+2.5 \%$ & $-0.7 \%$ \\
\hline NEQ, catalytic & 0.995 & 0.982 & 0.955 & 0.949 \\
& $-1.4 \%$ & $-0.0 \%$ & $+2.4 \%$ & $-0.8 \%$ \\
\hline \hline
\end{tabular}

Consequently, the atmospheric gas is non-reactive at the lower trajectory points with lower vehicle speeds and post-shock temperatures and t he chemistry is thus negligible. At the upper trajectory points, however, the temperatures are sufficient for the dissociation of a significant proportion of the molecules and the chemistry makes a difference.

The endothermic dissociation reactions act as a heat sink. Consequently, the translational temperatures behind the shock are lower if the gas chemistry is considered. Despite the reduced average molecular mass, this results in a reduced specific volume and thus higher density. The compression ratio over the shock is higher in case of chemical EQ and the shock stand-off distance is reduced. These correlations are clearly seen in figure 9a. While there is no difference between chemical EQ and the non-reacting gas solutions at trajectory points II and below, the shock stand-off distance at other trajectory points is higher in case of the non-reacting gas.

Chemical NEQ is important if the time scales of the flow field and the chemical reactions are of similar order. If the time scales of the chemical reactions are small compared to those of the convective flow, there is sufficient time for the reactions and the gas approaches local EQ. In that case, the differences between EQ and NEQ simulations are small and the surface catalysis has no influence as there are no catalytic effects possible in an EQ flow [27]. On the other hand, if the time scales of the chemical reactions are much higher than those of the convective flow, the gas can be considered chemically frozen and the calculation of the chemical NEQ is unnecessary. Unfortunately, the hot 
phase of the atmospheric entry usually lies in between these extremes and the chemical NEQ has a high influence, as is the case in this study. A good overview of the general simulation of the problem is given by Park [20]. Figure $9 \mathrm{~b}$ compares the temperature distribution and the carbon monoxide concentration of the EQ and NEQ calculations of trajectory point VIII with non-catalytic wall as boundary condition. In the NEQ flow, the gas composition does not jump to the local EQ. Instead, the gas composition and properties equal those of the non-reacting gas right after the shock. Afterwards, with ongoing reaction the gas properties transition towards those of the EQ solution. Thus, the gas properties at NEQ are between those of the non-reacting and the EQ gas. Consequently, the results of NEQ simulations can be expected to be between the non-reacting and the chemical EQ cases, which allows a rough estimation of the magnitude of the error introduced by the EQ assumption without the simulation of the NEQ.

Table 7 provides the pressure at positions according to those of the sensor location $P_{1}$ to $P_{4}$ on the wind tunnel model for these simulations. Figure 10 provides the pressure distribution on the front shield. The NEQ solutions for non-catalytic and fully catalytic walls lie between the solutions of the other gas models. Despite having a major impact on the heat flux, no effect of the catalytic activity on the pressure distribution is observed. The local reduction in the pressure trend of the EQ gas simulation (nominal case) originates from the sphere-cone-transition. This dent is only formed in

the case of the EQ solution since the shock stand-off distance only in this case is that small. This is a known phenomenon for similar aeroshell geometries, e.g. [28].

The influence of the chemistry decreases with the altitude of the respective trajectory point. While the pressures at the equivalent position $P_{1}$ to $P_{4}$ deviate up to $2.4 \%$ when comparing EQ and NEQ at trajectory point VIII, the difference drops to a maximum of $0.7 \%$ at trajectory point VII. It further decreases to $0.3 \%$ for trajectory point VI and finally becomes negligible at trajectory point V. This indicates, that the numerical results should be used cautiously for velocities above approximately $2 \mathrm{~km} / \mathrm{s}$, where the chemical NEQ has an influence on the pressure distribution.

\section{E. Comparison with Wind Tunnel Campaign Results}

The wind tunnel tests and their simulation as well as the simulation of the Mars entry for Mach 7.0 and below are obtained at identical Mach and Reynolds numbers. Despite this analogy, there 


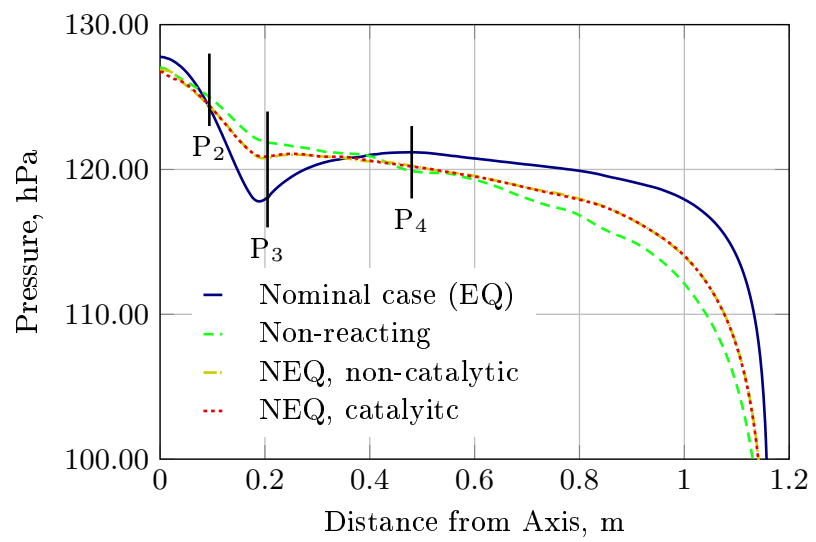

Fig. 10: Pressure distribution on the front for non-reacting gas, chemical EQ and NEQ at trajectory point VIII.
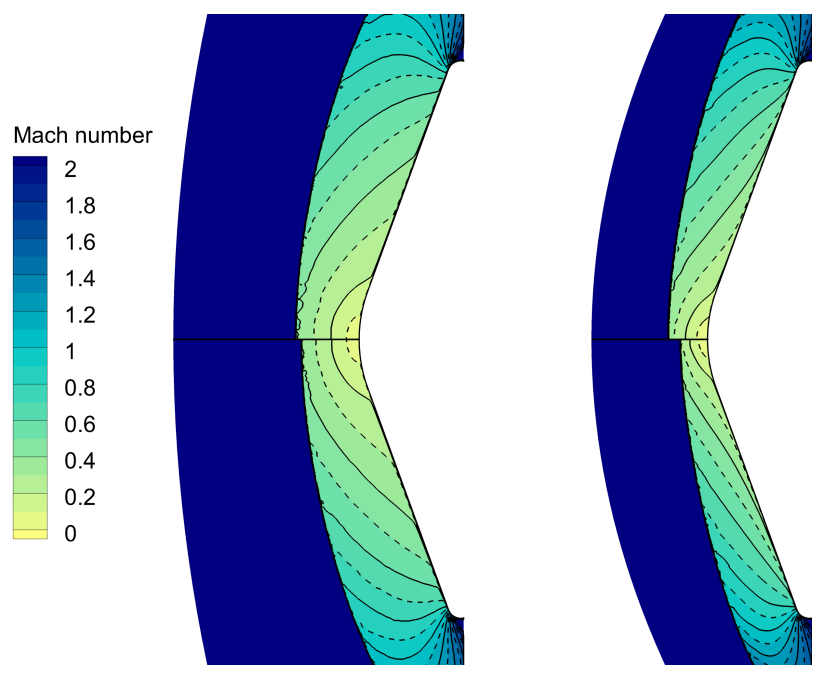

Fig. 11: Mach number distribution for Mach 5.0/5.3 (top) and Mach 7.0 (bottom) for the simulation of the wind tunnel experiments (left) and the flight simulation (right).

can be major differences in the flow. This is because the different boundary conditions, the variation of the gas and its properties, e.g. heat capacity ratio, as well as the chemistry in general are not reproduced by matching the Mach and Reynolds numbers. The different boundary conditions at the capsule surface mainly have an influence on the thin boundary layer. The impact on the surface pressure distribution is thus low. Also, the wind tunnel tests were performed at Mach 7.0 and below, so the corresponding trajectory points do not have the high temperatures needed for dissociation . This implies that the gas behaves similar to an ideal gas and has an almost constant heat capacity 


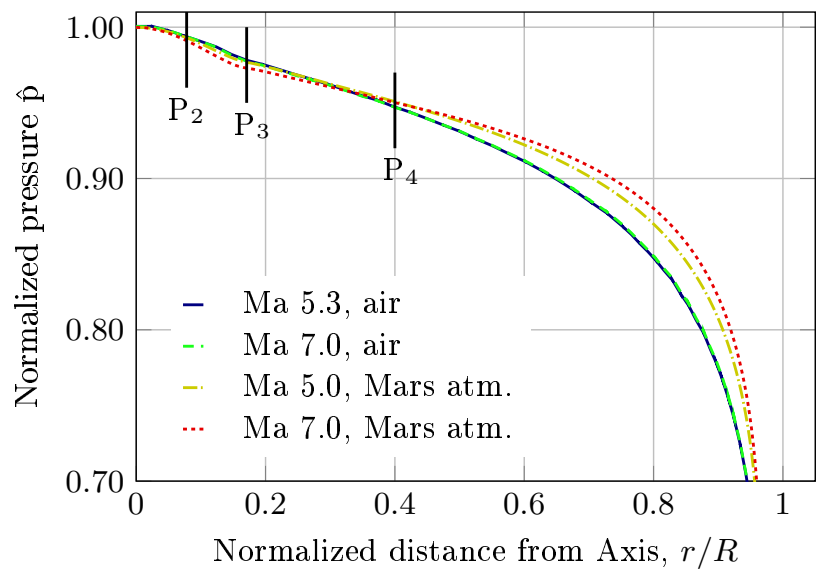

Fig. 12: Front surface pressure distribution of the numerical simulations of air and Mars atmosphere at Mach numbers 5.0/5.3 and 7.0 at $0^{\circ}$ AoA.

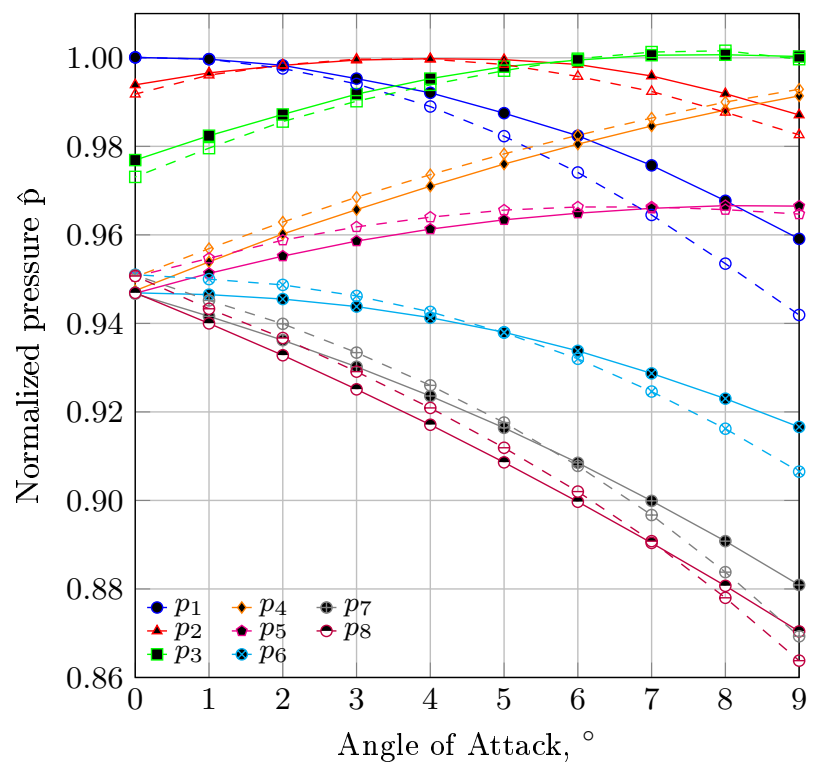

Fig. 13: Comparison of the numerical simulation of the wind tunnel tests (filled symbols, solid lines) and those of the Mars entry (empty symbols, dashed lines) at Mach number 7.0. All data normalized by equation 2 .

ratio. Consequently, the heat capacity ratio is the one significant factor that explains the differences between the simulated pressure distributions.

The heat capacity ratio of dry air is approximately $\gamma=1.4$ at the temperatures occurring in the wind tunnel experiments. Carbon dioxide, of which the Mars atmosphere mainly consists, has a lower heat capacity ratio as it is a three-atomic molecule. The value is approximately $\gamma=1.30$ 
at room temperature and decreases further with increasing temperatures. The heat capacity ratio varies with the temperature and chemical composition. It is subsequently not set to a constant value but calculated depending on the actual local gas composition and temperature. The real gas behaviour furthermore induces the variation of other gas properties, e.g. the viscosity and the mean molecular mass. The thermodynamic properties are calculated from partition functions or external lookup tables for each species in the gas [22].

The shock equations express the relation between heat capacity ratio and compression of the gas over the shock at a certain Mach number [29]. Lower heat capacity ratios lead to lower temperatures and higher densities after the shock. This reduces the shock stand-off distance, as seen in Fig. 11. The differences in the flow are higher when comparing the two gas models than for the two considered Mach numbers. In the numerical simulations of the wind tunnel experiments, the Mach number has a significantly lower impact and the shock stand-off distance is higher. It is consequently no surprise to see only small differences in the surface pressure distributions of the numerical wind tunnel experiments simulations in Fig. 12. In the simulations considering the atmosphere of Mars and real gas behaviour, the difference between the two Mach numbers is higher. This is due to the variation of the heat capacity ratio with temperature. At Mach 7.0, the temperatures behind the shock are higher and the heat capacity ratio is lower, which explains the greater reduction of the shock stand-off distance.

The pressure distribution variation is more pronounced in case of Martian atmosphere. Accordingly, the dependence of the pressure on the angle of attack is presented in Fig. 13 to show these differences. The lower shock stand-off distance of the EQ calculations makes the shock follow the surface more closely. The shock and also the surface pressure distribution are therefore more sensitive to changes in the angle of attack, which is manifested as higher gradients of the pressure trends with respect to the AoA. Therefore, it is necessary to consider the gas composition and temperatures when simulating atmospheric entry for FADS based reconstruction.

\section{Conclusions}

An experimental and numerical study has been carried out to provide reference data for the atmospheric reconstruction of the ExoMars 2016 lander Schiaparelli using the FADS approach. For 
this purpose, a scaled aerodynamic model of the capsule underwent extensive testing in DLR's hypersonic wind tunnel H2K. Tests allowed simultaniously duplicating the Mach and Reynolds numbers experienced during the entry flight . Since H2K can be operated only with air at total gas temperature up to $1100 \mathrm{~K}$, the chemistry of the entry environment could not be reproduced. Therefore, these tests were used for the validation of the DLR's CFD code TAU, which allowed simulating the Mars entry environment at selected points of the trajectory. The numerical approach has been adapted to the atmospheric conditions to provide information on the surface pressure distribution during entry. The potential influence of the assumptions regarding the flow turbulence and gas composition as well as the impact of the chemistry model have been analysed. Numerical results of flight conditions have been compared to those of the wind tunnel experiments simulations.

Wind tunnel tests have been conducted at three different Mach numbers for a total of 31 data points. The combination of a blunt body and the interest in low angles of attack means a high sensitivity to errors of the experimental set-up and required fastidious implementation. As a consequence, one focus of the study lay on the investigation and mitigation of experimental uncertainties. Despite the various measures taken to keep the uncertainties low, the observed stagnation pressures deviated from the theoretical expectations, so the wind tunnel data was normalized for the comparison with the numerical results. The results of the wind tunnel experiments and their numerical simulation then showed very good agreement and provided confidence for the simulation of the atmospheric entry.

The simulations conducted at flight conditions considered either chemical EQ in a 3D flow field, or chemical NEQ but in a $2 \mathrm{D}$ flow field. This saved computational resources, while allowing the quantification of the effect of assuming EQ chemistry. Furthermore, errors introduced by the assumptions regarding flow turbulence and atmospheric composition were investigated. It was found that the turbulence and gas composition have a low impact on the pressure distribution. However, a strong dependence of the solutions on the chemistry model has been revealed. At low trajectory points with velocities up to approximately $1 \mathrm{~km} / \mathrm{s}$, the gas is non-reacting and the influence of the chemistry can be neglected. Up to around $2 \mathrm{~km} / \mathrm{s}$, almost no difference between simulation with chemical EQ and with NEQ were found. However, the NEQ effects become more distinct at the 
trajectory points with higher gas total enthalpy and the surface pressure distributions gathered from EQ calculations should be used cautiously for vehicle speeds above $2 \mathrm{~km} / \mathrm{s}$, as the chemistry model in this case has an influence on the surface pressure distribution. The comparison of the numerical simulation of experiments and flight conditions shows the known strong influence of the heat capacity ratio on both, the shock stand-off distance and subsequently the shock geometry. Thus, this effects the absolute pressure values as well as the surface pressure distribution and is important for atmospheric and flow angle FADS reconstruction at the EDM flight conditions.

The investigation of the numerical uncertainties provided confidence in the numerical tool and the results of the entry flight simulations, especially for the lower part of the trajectory. Below $22 \mathrm{~km}$ at Mach 9.0 or $2 \mathrm{~km} / \mathrm{s}$, the numerical predictions and their assumptions were found to be very accurate. At higher altitudes and Mach numbers, the most significant error in the numerical predictions is due to the assumption of chemical EQ, reaching up to $2.4 \%$ deviation of the respective pressure distributions at Mach 14. The numerical pressure model will be used in Part II [12] to quantify the FADS performance along the Schiaparelli entry trajectory, concerning the reconstruction of atmospheric density, pressure, temperature, and Mach number, as well as the angle of attack and sideslip.

\section{References}

[1] Edquist, K. T., Dyakonov, A. A., Wright, M. J., and Tang, C. Y., "Aerothermodynamic Design of the Mars Science Laboratory Heatshield," AIAA Paper 2009-4075, doi:10.2514/6.2009-4075.

[2] Wright, M. J., Beck, R. A. S., Edquist, K. T., Driver, D., Sepka, S. A., Slimko, E. M., and Willcockson, W. H., "Sizing and Margins Assessment of Mars Science Laboratory Aeroshell Thermal Protection System," Journal of Spacecraft and Rockets, Vol. 51, No. 4, 2014, pp. 1125-1138.

[3] Little, A., Bose, D., Karlgaard, C., Munk, M., Kuhl, C., Schoenenberger, M., Antill, C., Verhappen, R., Kutty, P., and White, T., "The Mars Science Laboratory (MSL) Entry, Descent and Landing Instrumentation (MEDLI): Hardware Performance and Data Reconstruction," AAS Paper 13-078.

[4] Bose, D., White, T., Santos, J. A., Feldman, J., Mahzari, M., Olson, M., and Laub, B., "Initial Assessment of Mars Science Laboratory Heatshield Instrumentation and Flight Data," AIAA paper 2013-908. 
[5] Bose, D., White, T., Mahzari, M., and Edquist, K., "Reconstruction of Aerothermal Environment and Heat Shield Response of Mars Science Laboratory," Journal of Spacecraft and Rockets, Vol. 51, No. 4, 2014, pp. 1174-1184.

[6] Van Hove, B. and Karatekin, Ö., "Mars Atmosphere Reconstruction using a Flush Air Data System on the ExoMars Entry, Descent and Landing Demonstrator Module," in "10th International Planetary Probe Workshop," , 2013.

[7] Karlgaard, C. D., Kutty, P., Schoenenberger, M., Munk, M. M., Little, A., Kuhl, C. A., and Shidner, J., "Mars Science Laboratory Entry Atmospheric Data System Trajectory and Atmosphere Reconstruction," Journal of Spacecraft and Rockets, Vol. 51, No. 4, 2014, pp. 1029-1047.

[8] Schoenenberger, M., Van Norman, J., Karlgaard, C., Kutty, P., and Way, D., "Assessment of the Reconstructed Aerodynamics of the Mars Science Laboratory Entry Vehicle," Journal of Spacecraft and Rockets, Vol. 51, No. 4, 2014, pp. 1076-1093.

[9] Van Hove, B. and Karatekin, Ö., "Atmospheric Reconstruction with Stagnation Pressure Flight Data from Mars Science Laboratory," Journal of Spacecraft and Rockets, Vol. 54, No. 3, 2017, pp. 609-620.

[10] Portigliotti, S., Cassi, C., Montagna, M., Martella, P., Faletra, M., Boi, J., De Sanctis, S., Granà, D., Bayle, O., Blancquaert, T., and Lorenzoni, L., "EXOMARS 2016, the Schiaparelli Mission. EDL Demonstration Results from Real Time Telemetry before Unfortunate Impact," , 2017. 14th International Planetary Probes Workshop.

[11] Van Hove, B. and Karatekin, Ö., "Mars Entry, Descent, and Landing Reconstruction from Flight Data: Uncertainty Quantification," in "11th International Planetary Probe Workshop," , 2014.

[12] Van Hove, B., Karatekin, Ö., Schleutker, T., and Gülhan, A., "ExoMars FI ush Ai r Data System: Entry Simulation and At mospheric Reconstruction," Journal of Spacecraft and Rockets. Submitted.

[13] Niezgodka, F.-J., Der Hyperschallwindkanal H2K des DLR in Köln-Porz (Stand 2000) (German), DLRMitteilungen, Deutsches Zentrum für Luft- und Raumfahrt e. V., Köln, Germany, 2001.

[14] Tropea, C., Yarin, A., and Foss, J. F., Handbook of Experimental Fluid Mechanics, Vol. 1, Springer, 2007.

[15] Nitsche, W. and Brunn, A., Strömungsmesstechnik (German), VDI-Buch, Springer, 2006, doi:10.1007/3-540-32487-9.

[16] Schwamborn, D., Gerhold, T., and Heinrich, R., "The DLR TAU-code: Recent Applications in Research and Industry," in "Proceedings of the European Conference on Computational Fluid Dynamics," Delft, The Netherlands, 2006. 
[17] Langer, S., Schwöppe, A., and Kroll, N., "The DLR flow solver TAU-status and recent algorithmic developments," in "AIAA Paper 2014-0080," , 2014, doi:10.2514/6.2014-0080.

[18] CentaurSoft ${ }^{\mathrm{TM}}$, http://www.centaursoft.com.

[19] Menter, F. R., "Two-equation eddy-viscosity turbulence models for engineering applications," $A I A A$ Journal, Vol. 32, No. 8, 1994, pp. 1598âĂŞ-1605, doi: $10.2514 / 3.12149$.

[20] Park, C., Howe, J. T., Jaffe, R. L., and Candler, G. V., "Review of chemical-kinetic problems of future NASA missions, II: Mars entries," Journal of Thermophysics and Heat transfer, Vol. 8, No. 1, 1994, pp. 9-23.

[21] Fertig, M., "SACOMAR: Report and library on gas phase chemistry," SACOMAR Deliverable 6.3.

[22] Hannemann, K., Schramm, J., Wagner, A., Karl, S., and Hannemann, V., "A Closely Coupled Experimental and Numerical Approach for Hypersonic and High Enthalpy Flow Investigations Utilising the HEG Shock Tunnel and the DLR TAU Code," in "Aerothermodynamic Design, Review on Ground Testing and CFD," Sint-Genesius-Rode, Belgium, 2010.

[23] Wright, M. J., Prabhu, D. K., and Martinez, E. R., "Analysis of Apollo Command Module Afterbody Heating Part I: AS-202," Journal of Thermophysics and Heat Transfer, Vol. 20, No. 1, 2006, pp. 16-30, doi:10.2514/1.15873.

[24] Schneider, S. P., "Laminar-Turbulent Transition on Reentry Capsules and Planetary Probes," Journal of Spacecraft and Rockets, Vol. 43, No. 6, 2006, pp. 1153-1173.

[25] Neeb, D., Gülhan, A., and Merrifield, J. A., "Rough-Wall Heat Flux Augmentation Analysis Within the ExoMars Project," Journal of Spacecraft and Rockets, Vol. 53, No. 1, 2016, pp. 121-133, doi:10.2514/1.A33306.

[26] Park, C., "Injection-Induced Turbulence in Stagnation-Point Boundary Layers," AIAA Journal, Vol. 22, No. 2, 1984, pp. 219-225.

[27] Gnoffo, P. A., Weilmuenster, K. J., Hamilton, H. H., Olynick, D. R., and Venkatapathy, E., "Computational Aerothermodynamic Design Issues for Hypersonic Vehicles," Journal of Spacecraft and Rockets, Vol. 36, No. 1, 1999, pp. 21-43.

[28] Gnoffo, P. A., Weilmuenster, K. J., Braun, R. D., and Cruz, C. I., "Influence of Sonic-Line Location on Mars Pathfinder Probe Aerothermodynamics," Journal of Spacecraft and Rockets, Vol. 33, No. 2, 1996, pp. 169-177.

[29] Anderson, J. D., Fundamentals of Aerodynamics, Tata McGraw-Hill Education, 2011. 